\title{
O serviço de teleconsultoria assíncrona na APS: avaliação de uso e fatores associados do Programa Telessaúde Espírito Santo entre 2012 e 2015
}

\author{
The asynchronous teleconsulting service in the Primary Healthcare: use assessment and \\ correlated factors in the Espírito Santo Telehealth Programme between 2012 and 2015
}

\section{El servicio de teleconsultoría asíncrona en la APS: evaluación de uso y factores asociados del Programa Telesalud Espírito Santo entre 2012 y 2015}

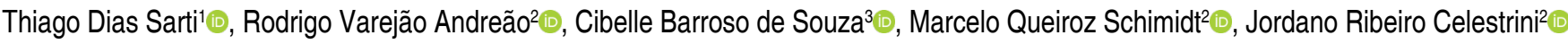 \\ ${ }^{1}$ Universidade Federal do Espírito Santo (UFES). Vitória, ES, Brasil. \\ ${ }^{2}$ Instituto Federal do Espírito Santo (IFES). Vitória, ES, Brasil. \\ ${ }^{3}$ Empresa Brasileira de Serviços Hospitalares, Hospital Universitário Walter Cantídio. Fortaleza, CE, Brasil.
}

\section{Resumo}

Introdução: As poucas avaliações de implementação de programas de telessaúde no Brasil mostram uma pequena utilização de seus serviços, devendo-se conhecer as razões disto. Objetivou-se analisar as taxas de utilização do serviço de teleconsultoria do Programa Telessaúde Espírito Santo no período de 2012 a 2015, verificando a influência de estratégias de fomento à sua utilização. Métodos: Estudo descritivo com base em dados secundários de perfil dos profissionais cadastrados e produção de teleconsultorias e webconferências. Resultados: No período analisado, foram 3076 profissionais cadastrados, sendo que 381 (12,4\%) realizaram 2182 consultorias $(41,4 \%$ solicitadas por enfermeiros), configurando uma utilização do serviço inferior ao seu potencial. Os dados sugerem que a utilização do serviço de teleconsultoria está associada à participação em seminários regionais (aumento de $15,5 \%$ no número de profissionais atuantes; $p=0,037$ ); que a gestão de cadastros dos profissionais tem impacto limitado; que há correlação positiva forte $(r=0,73 ; p=0,016)$ entre uso de teleconsultoria e de teleducação; e que a monitoria de campo pode influenciar positivamente. Conclusão: $O$ uso dos serviços de Telessaúde é pequeno, sendo que o foco de suas ações deva ser seu usuário final com vias a sua sustentabilidade.

Palavras-chave: Atenção Primária à Saúde; Telemedicina; Educação Continuada

Como citar: Sarti TD, Andreão RV, Souza CB, Schimidt MQ, Celestrini JR. O serviço de teleconsultoria assíncrona na APS: avaliação de uso e fatores associados do Programa Telessaúde Espírito Santo entre 2012 e 2015. Rev Bras Med Fam Comunidade. 2019;14(41):2068. https://doi.org/10.5712/rbmfc14(41)2068

\author{
Autor correspondente: \\ Thiago Dias Sarti. \\ E-mail: thiagosarti@yahoo.com.br \\ Fonte de financiamento: \\ Programa de Pesquisa para o SUS, Edital \\ FAPES/CNPq/Decit - SCTIE-MS/SESA \\ nํ0ㄴ 2015 - PPSUS, Fundação de Apoio \\ à Pesquisa do Espírito Santo (FAPES). \\ Parecer CEP: \\ 1.673 .424 (UFES), aprovado em \\ 10/08/2016. \\ Editor Convidado: \\ Airton Stein \\ Procedência e revisão por pares: \\ revisado por pares. \\ Recebido em: 23/04/2019. \\ Aprovado em: 11/05/2019.
}




\begin{abstract}
Introduction: The few implementation reviews in Brazil shows a small use of telehealth services and more research is required to know its causes. The main goal of this work is to analyze the use rate of the asynchronous teleconsulting service in the Espírito Santo Telehealth Programme from 2012 to 2015, looking to the influence of the different strategies employed to promote its use. Methods: This descriptive study takes into account secondary data of the health professionals registered and the services of teleconsulting and web conferences produced. Results: In this period, 3076 health professionals were registered, where 381 (12.4\%) requested 2182 teleconsulting $(41.4 \%$ of them were requested by nurses), which corresponds to a low use rate. After statistical analysis, it was observed that: the use rate of teleconsulting is influenced by the regional workshops (increase in $15.5 \%$ in the number of teleconsulting users; $p=0.037$ ); management of heath professional registration has a limited impact; there is a strong positive correlation $(r=0.73 ; p=0.016)$ between the use of the teleconsulting service and web conference participation; field monitoring may influence the use rate. Conclusion: Finally, this work confirms the low use rate of the Telehealth services, and recommends that centralizing the action plan at the end user is the key to promote its sustainability.
\end{abstract}

Keywords: Primary Health Care; Telemedicine; Education, Continuing

\title{
Resumen
}

Introducción: Las pocas evaluaciones de implementación de programas de telesalud en Brasil muestran una pequeña utilización de sus servicios, debiéndose conocer las razones de esto. Se pretendió analizar las tasas de utilización del servicio de teleconsultoría del Programa Telesalud Espírito Santo en el período de 2012 a 2015, verificando la influencia de estrategias de fomento a su utilización. Métodos: Estudio descriptivo basado en datos secundarios de perfil de los profesionales registrados y producción de teleconsultorías y webconferencias. Resultados: En el período analizado, fueron 3076 profesionales registrados, siendo que 381 (12,4\%) realizaron 2182 consultoras $(41,4 \%$ solicitadas por enfermeros), configurando una utilización del servicio inferior a su potencial. Los datos sugieren que la utilización del servicio de teleconsultoría está asociada a la participación en seminarios regionales (aumento del 15,5\% en el número de profesionales actuantes, $p=0,037$ ); que la gestión de los registros de los profesionales tiene un impacto limitado; que hay correlación positiva fuerte $(r=0,73 ; p=0,016)$ entre uso de teleconsultoría y de teleducación; y que el monitor de campo puede influir positivamente. Conclusión: El uso de los servicios de Telesalud es pequeño, siendo que el enfoque de sus acciones debe ser su usuario final con vías a su sustentabilidad.

Palabras clave: Atención Primaria de Salud; Telemedicina; Educación Continua

\section{Introdução}

AAtenção Primária à Saúde (APS) é a base para um sistema de saúde efetivo, eficiente, resolutivo e humanizado. ${ }^{1-3}$ Para isso, os profissionais que nela atuam devem estar adequadamente preparados. ${ }^{4}$ Contudo, a APS brasileira ainda enfrenta sérios e complexos desafios decorrentes, dentre outras coisas, de aspectos financeiros, políticos, culturais e gerenciais que envolvem o planejamento e a gestão do Sistema Único de Saúde (SUS). ${ }^{5}$ E um dos principais desafios enfrentados atualmente reside exatamente na qualificação profissional para a APS, com vistas a sua maior qualidade. ${ }^{6}$

É neste sentido que foi criado em 2010 o Programa Telessaúde Brasil, sendo este ampliado e redefinido como Programa Nacional Telessaúde Brasil Redes (PNTBR) em 2011, tendo como objetivo o fortalecimento da APS brasileira. ${ }^{7}$ Induzido e fomentado pelo Ministério da Saúde, em parceria com outros ministérios e instituições de ensino superior (IES) e gestões estaduais e municipais de saúde, o Telessaúde disponibiliza tecnologias e ferramentas de informação e comunicação (TIC) que criam canais de comunicação da APS com outros níveis de atenção à saúde, estabelecendo processos de educação permanente que incluem discussão de casos clínicos, palestras, acesso a materiais educativos, atualização clínica e realização de procedimentos diagnósticos (exemplo: ECG). ${ }^{7-9}$

O PNTBR é executado de maneira descentralizada a partir de Núcleos Estaduais de Telessaúde vinculados a IES e gestões estaduais e municipais de saúde, concentrando suas ações geralmente em torno de três modalidades de serviços: teleconsultorias assíncrona e síncrona, no qual os profissionais da APS 
enviam problemas oriundos de seu cotidiano para solução compartilhada com especialistas alocados nos núcleos; teleducação, compreendendo palestras virtuais, materiais educativos, atualizações clínicas, etc.; e telediagnóstico, com grande variação de exames complementares realizados pelos distintos núcleos de telessaúde. ${ }^{7}$ Tais ações têm o potencial, por exemplo, de reduzir a taxa de encaminhamentos de pacientes da APS para outros níveis de atenção, garantir acesso a serviços de saúde em locais de difícil acesso e reduzir os custos da atenção à saúde. $8,10,11$

O Programa Telessaúde Espírito Santo (ES), fruto de parcerias entre duas instituições de ensino federais e a Secretaria de Estado da Saúde do ES, iniciou suas atividades em 2012 oferecendo serviços de consultoria e teleducação a mais de 60 municípios do ES. ${ }^{9}$ Desde seu início, o desafio de fomentar o uso das ferramentas pelos profissionais de saúde da APS do ES sempre esteve presente. A baixa utilização de consultorias e teleducação é um problema de todos os Núcleos de Telessaúde do Brasil, ${ }^{10}$ não havendo ainda estratégias bem consolidadas e avaliadas para reverter este quadro.

São poucos os trabalhos publicados até o momento que avaliam os fatores relacionados à utilização dos serviços disponibilizados pelos programas de Telessaúde. ${ }^{8,10,11}$ Alguns fatores interferem na utilização das consultorias e ações de teleducação, como a percepção do profissional sobre o potencial positivo das ferramentas do Telessaúde, a organização dos serviços de saúde, o acesso a computadores e internet de qualidade, a fixação dos profissionais da APS em seus locais de trabalho, o suporte ao uso das tecnologias, a familiaridade, capacidade e autonomia no uso das ferramentas virtuais e a dinâmica da gestão municipal e estadual em saúde. ${ }^{10}$

Desde sua implantação, o Telessaúde ES vem testando estratégias para aumentar o uso de seus serviços, em particular os de teleconsultoria assíncrona. ${ }^{9}$ Dentre essas estratégias, citam-se monitorias de campo, com contato permanente com gestões municipais e profissionais de saúde via ligações telefônicas e seminários regionais com participação ativa dos profissionais de saúde e gestores. Por outro lado, não se sabe o grau de influência mútua que os serviços de teleconsultoria e teleducação têm em seu uso.

É neste sentido que o presente trabalho visa apresentar os dados gerais de utilização dos serviços de teleconsultoria do Programa Telessaúde ES, bem como analisar a possível influência da monitoria de campo e dos seminários regionais no padrão de utilização das teleconsultorias. Além disso, testa-se a hipótese de correlação entre as taxas de uso das teleconsultorias e ações de teleducação (webconferências). Os resultados deste trabalho podem contribuir nas estratégias a serem adotadas pelos Núcleos de Telessaúde com respeito à promoção de seus serviços e a garantia de sua sustentabilidade.

\section{Métodos}

Trata-se de um estudo retrospectivo, de natureza quantitativa, de análise de dados secundários oriundos dos cadastros de profissionais de saúde e dos bancos de dados de utilização dos serviços prestados pelo Programa Telessaúde Espírito Santo entre 2012 e 2015. Os dados utilizados referem-se ao perfil profissional dos cadastrados no programa e à taxa de utilização dos serviços de consultorias e webconferências prestados pelo Telessaúde ES. Os profissionais de saúde foram categorizados em três grupos distintos, tomando como base seu cadastro no programa: (1) profissionais cadastrados antes de 2015 sem atualização do mesmo em 2015; (2) profissionais cadastrados antes de 2015 com atualização deste em 2015; e (3) profissionais cujo primeiro cadastro no programa ocorreu em 2015. 
Com esta categorização, busca-se avaliar a influência da gestão do cadastro profissional no programa na sua utilização. O cadastro do profissional consiste em uma série de dados englobando aspectos demográficos e profissionais. Com o cadastro, o profissional recebe os dados que dão acesso à plataforma virtual de consultorias do Telessaúde ES: Plataforma Salus (telessaude.ifes.edu.br/salus). As ações de webconferência (palestras virtuais) não exigem cadastro e estão abertas à comunidade de profissionais de saúde no site do programa.

Inicialmente, foram realizadas análises temporais das taxas de utilização dos serviços de consultoria entre a implantação do programa em 2012 até o ano de 2015. A partir destas análises, foram realizadas associações estatísticas entre taxas de utilização dos serviços de consultorias e webconferência e perfil de cadastro dos profissionais de saúde com os dados de 2015 , tendo em vista que foi neste ano que o uso das ferramentas do programa alcançou patamar significativo e minimamente estável.

Os dados foram analisados por medidas descritivas simples e as correlações entre taxas de utilização dos serviços e perfil de cadastro do profissional foram feitas com o Coeficiente de Correlação de Spearman, considerando-se correlações estatisticamente significativas aquelas com $p \leq 0,05$.

Além disso, verificou-se possível associação entre a realização de Seminários Regionais de Telessaúde no ES e a taxa de utilização dos serviços de teleconsultoria assíncrona. Os seminários regionais são encontros realizados em uma dimensão regional, conforme o Plano Diretor de Regionalização em Saúde do Espírito Santo, com o intuito de sensibilizar e fomentar o uso das ferramentas do programa.

Foram organizados em parceria com as superintendências regionais de saúde vinculadas à Secretaria Estadual de Saúde do estado, contando com a participação das gestões municipais e profissionais da APS dos municípios. Nestes eventos, ocorrem capacitações para uso das ferramentas e palestras organizadas pela equipe gestora do programa. Foram realizados quatro seminários, um em cada macrorregião de saúde do ES, entre abril e junho de 2014.

Nesta análise, foram selecionados os profissionais de saúde que participaram dos seminários regionais que estavam cadastrados no Telessaúde há pelo menos 6 meses da data de realização do evento. Comparou-se então o número de consultorias realizadas por esses profissionais nos seis meses anteriores ao evento com o número de consultorias realizadas no semestre posterior ao seminário, utilizando-se para isto do teste Qui-quadrado com nível de significância de 5\%.

Todas as análises estatísticas foram feitas com o auxílio dos software SPSS 20.0 (Statistical Package for the Social Science). O projeto foi aprovado pelo Comitê de Ética em Pesquisa do Centro de Ciências da Saúde - Universidade Federal do Espírito Santo, com o parecer 700.093/2014.

\section{Resultados}

Em 2015, o Telessaúde ES atingiu 3076 profissionais cadastrados, predominando profissionais que atuam em municípios da Região de Saúde Metropolitana, que agrega a Região Metropolitana de Vitória e alguns municípios do interior do ES. Agentes Comunitários de Saúde $(28,4 \%)$ e médicos $(23,4 \%)$ predominam no programa (Tabela 1). 
Tabela 1. Perfil dos profissionais de saúde cadastrados no Programa Telessaúde Espírito Santo - Espírito Santo, 2015.

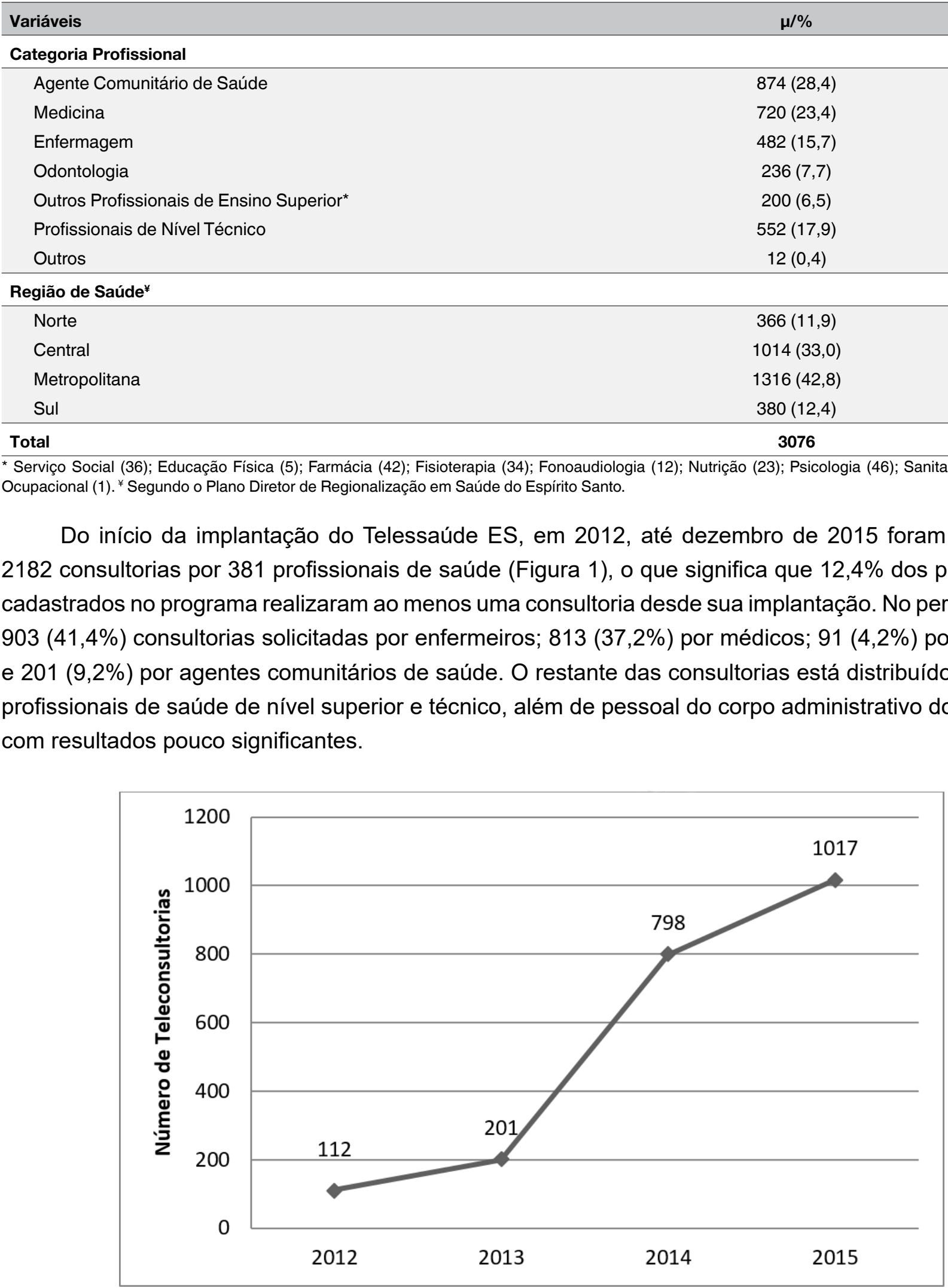

Figura 1. Teleconsultorias assíncronas solicitadas por ano no Programa Telessaúde ES, Espírito Santo, 2012-2015. 
A Figura 2 mostra o quantitativo de consultorias solicitadas entre 2012 e 2015 distribuídas por mês do ano. Nos dois primeiros anos do programa, observa-se um número muito baixo de solicitações, com eventuais picos de utilização, em particular no mês de outubro de 2013, logo seguido por uma queda acentuada do quantitativo de consultorias nos meses seguintes. Este cenário é revertido em meados de 2014, sustentando-se um número maior de consultorias solicitadas durante o restante de 2014 e todo o ano de 2015.

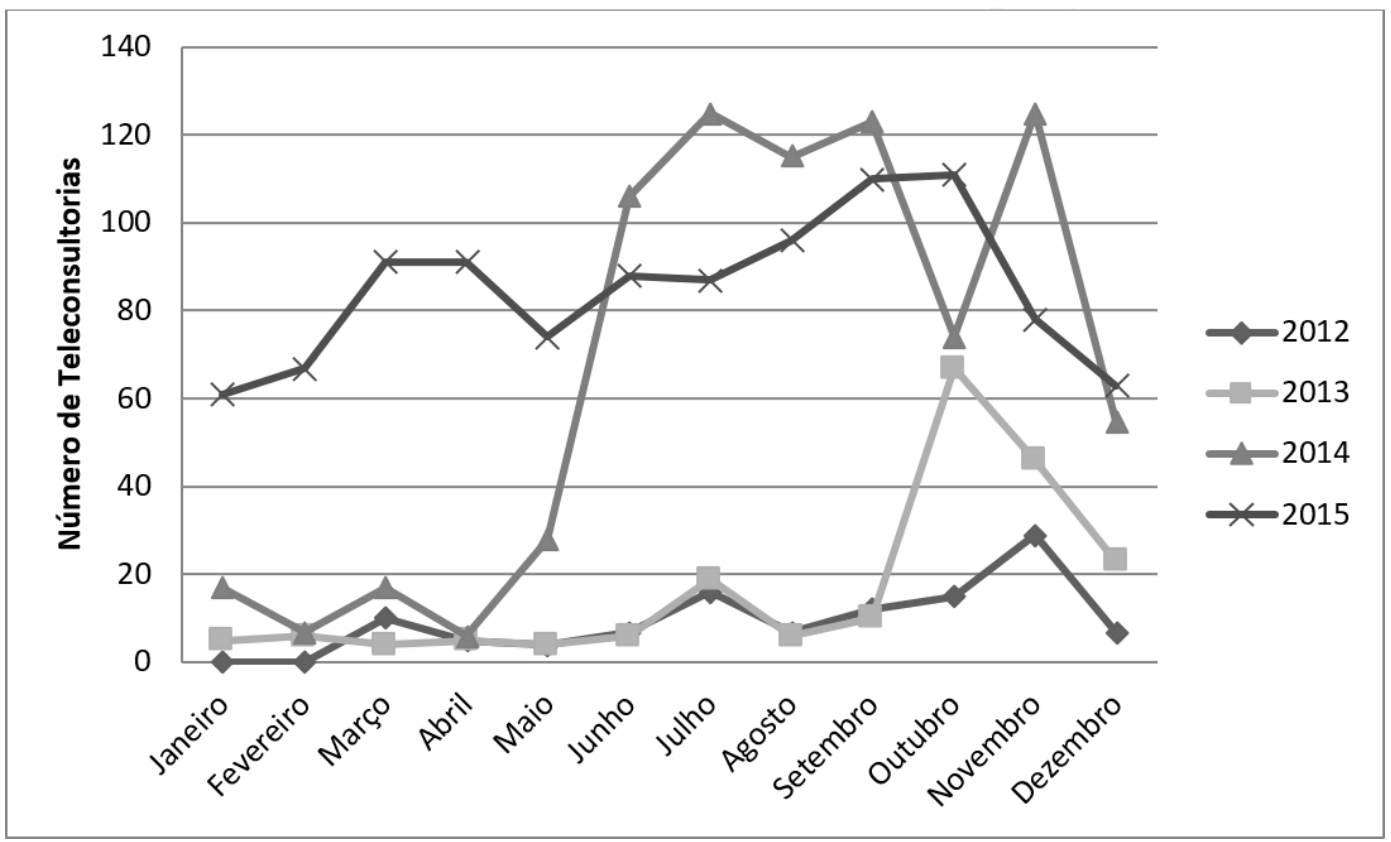

Figura 2. Teleconsultorias assíncronas solicitadas pelos profissionais de saúde distribuídas por mês do ano no Programa Telessaúde ES, Espírito Santo, 2012-2015.

O pico de consultorias observado em outubro de 2013 ocorreu após um movimento no qual um membro da equipe do programa realizou ligações telefônicas para uma amostra dos profissionais cadastrados com o objetivo de sensibilizá-los para o uso das ferramentas. Este movimento foi pontual e com resultados restritos, como se depreende da queda acentuada e sustentada do número de consultorias nos meses seguintes.

Por outro lado, o súbito aumento das consultorias a partir de 2014 coincide temporalmente com a realização dos Seminários Regionais em Telessaúde no âmbito da APS. Ao todo, cem profissionais de saúde participaram dos seminários, sendo que 45 estavam cadastrados no programa há pelo menos 6 meses da data do evento. Com base nos números de consultorias realizadas por estes 45 profissionais, segundo o critério de inclusão nesta análise, constatou-se que a taxa média de utilização nos 6 meses anteriores e posteriores ao evento foi de 0,6 e 1,0 consultoria por profissional, respectivamente.

Isto significa que antes do seminário apenas 9 (20\%) profissionais realizaram pelo menos uma consultoria (máximo de 6 consultorias por profissional), enquanto que após o seminário este número foi de 16 (35,5\%), com um máximo de 9 consultorias por profissional, sendo esta uma diferença com significância estatística (valor-p=0.037), o que sugere uma associação entre a realização de seminários regionais de fomento ao uso das ferramentas de Telessaúde e uma maior taxa de utilização de consultorias assíncronas. 
Ainda com base nos dados apresentados na Figura 2, apesar desta série histórica limitada e irregular, é possível aventarmos a hipótese de que o quantitativo de consultorias sofre significativa queda entre os meses de dezembro e fevereiro de cada ano, talvez em decorrência de período de férias, sendo algo a ser mais bem estudado em outros trabalhos.

Especificamente no ano de 2015, foram realizadas 1023 consultorias via Plataforma Salus. Destas, tomando por base o cadastro do profissional no Telessaúde ES, $678(66,3 \%)$ consultorias foram solicitadas por profissionais cadastrados antes de 2015 e sem atualização naquele ano e $345(33,7 \%)$ por profissionais cadastrados em 2015 (novos cadastros naquele ano). Nenhuma consultoria foi solicitada por profissionais cadastrados antes de 2015 , cujos cadastros foram atualizados naquele ano.

Este total de consultorias foi solicitado por 207 profissionais, sendo $74(35,7 \%)$ com cadastros novos em 2015 e 133 (64,3\%) com cadastros anteriores a 2015 sem atualização neste ano. Em relação ao total de profissionais cadastrados no Telessaúde ES até o final de 2015, são 551 (17,9\%) com cadastros novos realizados em 2015, 1376 (44,7\%) cadastrados antes de 2015 e atualizados neste ano e 1153 (37,4\%) profissionais com cadastros anteriores a 2015 sem atualização. Ou seja, 13,4\% dos profissionais com cadastros novos em 2015 e 11,5\% dos profissionais cadastrados antes de 2015 realizaram ao menos uma consultoria ao longo do ano.

Por outro lado, identificou-se que não há correlação entre o número de consultorias realizadas em 2015 e o fato do profissional ser cadastrado antes de 2015 com atualização neste ano ( $r=0,37 ; p=0,27)$. Contudo, encontrou-se correlação positiva forte entre o profissional ter sido cadastrado em 2015 e o número de consultorias quando se correlaciona o cadastro em um determinado mês com o número de consultorias solicitadas no mês seguinte $(r=0,78 ; p=0,004)$.

Desta forma, no geral observa-se que uma pequena parcela dos profissionais cadastrados no Telessaúde mantêm-se ativos no serviço de teleconsultoria (algo em torno de 11 a 13\% nesta amostra), sendo que alguns estabelecerão um bom vínculo temporal com o programa, não necessitando de constantes atualizações cadastrais como forma de sensibilização para a participação no programa.

Assim, os dados deste estudo sugerem que a estratégia de atualização do cadastro de profissionais pouco ou nada influencia na utilização de consultorias. Por outro lado, a proximidade temporal com o cadastro realizado pelo profissional parece aumentar a probabilidade de participação ativa no serviço de consultoria para uma parcela de usuários, sendo necessário um contínuo esforço dos Programas de Telessaúde para a incorporação de novos profissionais entre os usuários.

Buscou-se também identificar possível correlação entre a participação profissional nas webconferências e o número de consultorias solicitadas, verificando a hipótese destes serviços se influenciarem na sua utilização. A análise de correlação feita para distintas categorias profissionais (médicos, enfermeiros, etc.) ao longo do ano não foi estatisticamente significativa.

No entanto, quando se correlaciona a participação em webconferência tomando globalmente as categorias profissionais juntas em determinado mês com o número de consultorias solicitadas por todas as categorias profissionais no mesmo mês, encontra-se uma forte correlação global positiva entre estas variáveis ( $r=0,73 ; p=0,016)$, com a exceção do mês de abril de 2015 (Tabela 2). 
Tabela 2. Correlação entre participação em webconferência e solicitação de teleconsultorias no Programa Telessaúde ES - Espírito Santo, 2015.

\begin{tabular}{lccc}
\hline Mês - webconferência & Mês - teleconsultoria & $\mathbf{r}^{*}$ & $\mathbf{p}$ \\
\hline Janeiro & Janeiro & 0,84 & 0,002 \\
Fevereiro & Fevereiro & 0,75 & 0,012 \\
Março & Março & 0,63 & 0,050 \\
\hline Abril & Abril & 0,47 & 0,167 \\
Maio & Maio & 0,81 & 0,004 \\
Junho & Junho & 0,95 & 0,000 \\
Julho & Julho & 0,91 & 0,000 \\
Agosto & Agosto & 0,90 & 0,000 \\
Setembro & Setembro & 0,96 & 0,000 \\
Outubro & Outubro & 0,78 & 0,008 \\
Novembro & Novembro & 0,81 & 0,005 \\
Dezembro & Dezembro & 0,73 & 0,017 \\
Janeiro - Dezembro & Janeiro-Dezembro & 0,73 & 0,016 \\
\hline
\end{tabular}

* Coeficiente de Correlação de Sperman.

No intuito de entender esse padrão anômalo observado no mês de abril de 2015, percebeu-se que a participação de psicólogos e agentes comunitários de saúde (ACS) nas webconferências neste mês foi acima da média dos meses imediatamente anteriores e posteriores, o que não aconteceu nas consultorias. Uma análise igualando a participação dos psicólogos e agentes comunitários de saúde no mês de abril/2015 com as médias de participação histórica destes profissionais nas webconferências também não encontrou correlação estatisticamente significativa $(r=0,65 ; p=0,067)$.

No entanto, quando essa análise é feita em conjunto para as duas categorias profissionais, encontra-se uma correlação forte positiva e significativa entre webconferência e consultorias neste mês $(r=0,91 ; p=0,005)$, evidenciando que a exceção observada em abril de 2015 deve-se a uma maior participação destas duas categorias nas webconferências do mês, contrastando com as médias históricas do restante do ano.

Apesar de não ser possível controlar todas as variáveis que podem explicar esse comportamento discrepante, sugere-se que o conteúdo das webconferências tenha influenciado a maior participação de psicólogos e ACSs, já que neste mês houve um maior enfoque na abordagem da violência na APS, sendo esta uma temática que faz múltiplas interfaces com o trabalho destes profissionais e que possivelmente aumente seu interesse e participação.

\section{Discussão}

Os dados apresentados neste trabalho mostram que a utilização das ferramentas oferecidas pelo Telessaúde no Espírito Santo está abaixo de seu potencial, o que exige respostas no sentido de aproximar os profissionais da APS das TICs. Esta é uma realidade comum aos demais Núcleos de Telessaúde de todo o Brasil, $, 10,12,13$ não sendo diferente o padrão observado no ES, como mostrado neste e em outros trabalhos. ${ }^{14}$ 
Fatores relacionados ao profissional de saúde e ao contexto assistencial influenciam esta taxa de utilização dos serviços de Telessaúde..$^{8,10,13} \mathrm{O}$ processo de incorporação das tecnologias de informação no cotidiano dos serviços de saúde é complexo e dinâmico, sendo ainda pouco explorado na literatura. Dentre outros fatores, pode-se dizer que a incorporação das TICs nos processos de trabalho cotidianos dos profissionais depende da familiaridade destes com as tecnologias e da avaliação que os mesmos fazem da potencialidade que estas ferramentas têm para contribuir para a qualificação da atenção à saúde.

Em um contexto assistencial de precariedade estrutural, sobrecarga de trabalho e informatização insuficiente, como é o caso da APS brasileira, a aceitabilidade e usabilidade destas tecnologias por profissionais e usuários dos serviços podem ser prejudicadas. ${ }^{10,15}$

Tem-se, então, o desafio de adequar as tecnologias ao contexto e perfil de seus potenciais usuários, disponibilizando informações de qualidade a respeito de seus limites e potencialidades para a melhoria da atenção à saúde. Igualmente fundamentais são os esforços e investimentos dos gestores de todo o sistema de saúde no sentido de melhorar a estrutura e a integração dos serviços; de capacitar os profissionais na incorporação destas tecnologias em seu cotidiano de trabalho; e de promover a fixação dos profissionais em seus locais de trabalho.

Por sua vez, o perfil dos profissionais solicitantes de consultorias no ES, no qual predominam enfermeiros e médicos, está de acordo com o observado em outros cenários, ${ }^{10-12}$ sendo que a alta participação de Agentes Comunitários de Saúde em teleconsultorias observada no Núcleo do Rio Grande do Sul ${ }^{12}$ não se reproduziu no ES, o que aponta para um importante aspecto a ser trabalhado no sentido de promover o uso das ferramentas de teleconsultoria.

A forte concentração de teleconsultorias solicitadas por enfermeiros e médicos deve ser vista, por um lado, como um movimento positivo na medida em que estes profissionais ocupam um espaço central nas equipes de saúde da APS, embora isso possa representar uma menor participação dos demais profissionais de saúde na dinâmica de cuidado dos usuários dos serviços de saúde. Quer dizer, este perfil de uso pode estar relacionado a um processo de trabalho médico e enfermeiro centrado possivelmente problemático para o perfil de organização da APS no Brasil.

O forte e sustentado crescimento do número de teleconsultorias observado a partir de meados de 2014 (Figuras 1 e 2) está temporalmente associado à realização dos seminários regionais de Telessaúde. Além disso, um pico pontual de uso das consultorias observado no final de 2013 seguiu-se a uma estratégia de contato próximo e sensibilização dos profissionais cadastrados no Telessaúde. Isso reforça a necessidade de implementação de estratégias de monitoramento e suporte contínuos aos municípios e profissionais de saúde no que diz respeito à implantação do Telessaúde..$^{9,16}$

A realização de seminários e outros tipos de eventos técnicos realizados em locais o mais próximo possível da realidade dos profissionais de saúde, bem como a consolidação de monitorias de campo, com produção de dados sobre a utilização das TICs e contato permanente com gestores e profissionais de saúde, ${ }^{14}$ tornam-se então estratégias a serem consideradas e melhor avaliadas devido à suas potencialidades evidenciadas neste trabalho. E para que isso possa ocorrer a contento, o dimensionamento das equipes técnicas ligadas aos Núcleos de Telessaúde deve ser bem planejada, o que traz à tona o tema do adequado financiamento destes programas no nível local. 
Outra estratégia de sensibilização para o uso das TICs oferecidas pelo Telessaúde ES avaliada neste trabalho foi a atualização dos cadastros dos profissionais de saúde. Em 2015, viu-se que muitos profissionais cadastrados não mais utilizavam ou nunca haviam utilizado as tecnologias. Um dos motivos aventados era o esquecimento do programa, motivo pelo qual uma atualização de cadastro poderia ser um passo para uma reaproximação do profissional com o programa.

Contudo, essa atualização não trouxe resultados. O provável motivo é que esta atualização ficou sob a responsabilidade do diretor do serviço de saúde e não do profissional de saúde. O diretor coletava as informações com os profissionais e encaminhava os cadastrados atualizados para a coordenação do Telessaúde ES, ou seja, o processo era feito sem um movimento ativo do profissional que viria em tese utilizar o serviço.

Estando esta hipótese correta, pode-se afirmar que esta estratégia não é produtiva, sendo fundamental centralizar a relação do programa diretamente com o profissional de saúde, visto que contatos próximos a estes impactam positivamente na utilização das ferramentas de Telessaúde. Gestores e diretores de serviços devem estar próximos de todo o processo de construção e implementação de programas de Telessaúde,${ }^{9}$ mas não podem ser os únicos intermediários na relação com os profissionais de saúde, correndo-se o risco de insucessos no caso de gestões fragilizadas.

Tudo isso é comprovado pelo fato dos profissionais cadastrados recentemente (em 2015), quando o Telessaúde ES passou a atuar de maneira mais próxima das equipes de saúde e com menor dependência da gestão municipal como intermediária, participarem mais das teleconsultorias. Reforça a hipótese o achado de que a participação dos profissionais nos seminários regionais influencia positivamente sua utilização do Telessaúde.

Em relação à hipótese de teleconsultorias e teleducação se fortalecerem mutuamente, mostrou-se que há correlação positiva entre ambos os serviços, embora não tenha sido possível avaliar o impacto de cada serviço na taxa de utilização do outro, sendo este um dado não explorado por outras publicações. Isso aponta para a importância da diversificação e fortalecimento das ações realizadas pelos Núcleos de Telessaúde.

\section{Considerações finais}

A importância das TICs no fortalecimento dos sistemas de saúde e da APS, apesar de grandes lacunas ainda existentes na literatura científica da área, aponta para a necessidade de fortalecimento do Programa Telessaúde Brasil Redes e suas experiências locais. A baixa utilização dos serviços de Telessaúde observado em todo o país, o que ocorre também no ES, exige investimentos em pesquisas e ações que busquem conhecer os fatores que se relacionam à utilização das TICs nos distintos cenários da saúde.

Neste trabalho, sugere-se que investimentos na estruturação dos serviços de saúde, na adequação das tecnologias à realidade dos profissionais de saúde e, mais importante, uma maior proximidade dos Programas de Telessaúde locais com os profissionais de saúde dos serviços, com menor dependência de intermediários dos níveis de gestão nesta relação, são essenciais para a consolidação destas tecnologias no Brasil. Sugere-se também a realização de seminários regionais e movimentos de permanente contato com os profissionais como estratégias essenciais para todos os Núcleos de Telessaúde do país.

\section{Agradecimentos}

À Fundação de Apoio à Pesquisa do Espírito Santo (FAPES) pelo suporte financeiro através do Programa de Pesquisa para o SUS conforme Edital FAPES/CNPq/Decit -SCTIE-MS/SESA nº 05/2015 - PPSUS. 


\section{Contribuição dos autores}

Concepção e delineamento do estudo: TDS, RVA. Aquisição, análise e interpretação dos dados: TDS, RVA, CBS, MQS, JC. Redação preliminar: TDS. Revisão crítica da versão preliminar: RVA, CBS, MQS, JC. Todos os autores aprovaram a versão final e concordaram com prestar contas sobre todos os aspectos do trabalho.

\section{Conflito de interesses}

Declaram não haver.

\section{Referências}

1. Jerant A, Fenton JJ, Franks P. Primary care attributes and mortality: a national person-level study. Ann Fam Med. 2012;10(1):34-41. https://doi.org/10.1370/afm.1314

2. World Health Organization (WHO). The world health report 2008: primary health care now more than ever. Geneva: WHO; 2008.

3. Macinko J, Starfield B, Shi L. The contribution of primary care systems to health outcomes within Organization for Economic Cooperation and Development (OECD) countries, 1970-1998. Health Serv Res. 2003;38(3):831-65. https://doi.org/10.1111/1475-6773.00149

4. Starfield B. Atenção Primária: equilíbrio entre necessidades de saúde, serviços e tecnologia. Brasília: UNESCO, Ministério da Saúde; 2002.

5. Costa NR. A Estratégia de Saúde da Família, a atenção primária e o desafio das metrópoles brasileiras. Ciênc Saúde Coletiva. 2016;21(5):1389-98. https://doi.org/10.1590/1413-81232015215.24842015

6. Arantes LJ, Shimizu HE, Merchán-Hamann E. Contribuições e desafios da Estratégia Saúde da Família na Atenção Primária à Saúde no Brasil: revisão da literatura. Ciênc Saúde Coletiva. 2016;21(5):1499-510. https://doi.org/10.1590/1413-81232015215.19602015

7. Haddad AE. Experiência Brasileira do Programa Nacional Telessaúde Brasil. In: Mathias I, Monteiro A, orgs. Gold Book: inovação tecnológica em educação e saúde. Rio de Janeiro: UERJ; 2012. p. 12-44.

8. Soriano Marcolino M, Minelli Figueira R, Pereira Afonso Dos Santos J, Silva Cardoso C, Luiz Ribeiro A, Alkmim MB. The Experience of a Sustainable Large Scale Brazilian Telehealth Network. Telemed J E Health. 2016;22(11):899-908. https://doi.org/10.1089/tmj.2015.0234

9. Sarti TD, Andreão RV, Schimidt MQ, Celestrini J. Teleconsultoria assíncrona como ferramenta de suporte ao trabalho em saúde. Rev Bras Pesq Saúde. 2013;15(3):79-84.

10. Alkmim MB, Marcolino MS, Figueira RM, Sousa L, Nunes MS, Cardoso CS, et al. Factors associated with the use of a teleconsultation system in Brazilian primary care. Telemed J E Health. 2015;21(6):473-83. https://doi.org/10.1089/tmj.2014.0112

11. Marcolino MS, Alkmim MB, Assis TGP, Sousa LAP, Ribeiro ALP. Teleconsultorias no apoio à atenção primária à saúde em municípios remotos no estado de Minas Gerais, Brasil. Rev Panam Salud Publica. 2014;35(5/6):345-52.

12. Harzheim E, Siqueira AC, Correa APB, Detoni Filho A, Katz N. Overview of teleconsultations after deployment of telehealth platform in RS. In: Anais da 12th Wonca World Rural Health Conference; 2014; Gramado, RS, Brasil.

13. Oliviera DG, Frias PG, Vanderlei LCM, Vidal SA, Novaes MA, Souza WV. Análise da implantação do Programa Telessaúde Brasil em Pernambuco, Brasil: estudo de casos. Cad Saúde Pública. 2015;31(11):2379-89.

14. Garcia ACP, Andrade MAC, Zandonade E, Prado TN, Freitas PSS, Cola JP, et al. Análise da organização da Atenção Básica no Espírito Santo: (des)velando cenários. Saúde Debate. 2014;38(no.spe):221-36.

15. Harzheim E, Gonçalves MR, Umpierre RN, da Silva Siqueira AC, Katz N, Agostinho MR, et al. Telehealth in Rio Grande do Sul, Brazil: Bridging the Gaps. Telemed J E Health. 2016;22(11):938-44. https://doi.org/10.1089/tmj.2015.0210

16. Harzheim E, Correa APB, Siqueira AC, Melo LN, Katz N. Support to the implementation of the new TelessaúdeRS centers telehealth. In: Anais da 12th Wonca World Rural Health Conference; 2014; Gramado, RS, Brasil. 